\title{
Estructura de un bosque natural perturbado de Pinus tropicalis Morelet en Galalón, Cuba
}

\author{
María A. Valdés-Sáenz ${ }^{1}$, Yudel García-Quintana ${ }^{1,4}$, Antonio Escarré-Esteve ${ }^{2}$, Joel Flores ${ }^{3}$, \\ Gretel Geada-López ${ }^{1}$, Yasiel Arteaga-Crespo ${ }^{1}$ y Carmen R. Valdés-Sáenz ${ }^{1}$ \\ 'Facultad de Forestal y Agronomía, Universidad de Pinar del Río, Pinar del Río, Cuba \\ ${ }^{2}$ Universidad de Alicante, Alicante, España \\ ${ }^{3}$ División de Ciencias Ambientales, Instituto Potosino de Investigación Científica y Tecnológica, A.C., \\ San Luis Potosí, México \\ ${ }^{4}$ Autor para la correspondencia: ygarcia@af.upr.edu.cu
}

\begin{abstract}
Resumen: Los bosques naturales de Pinus tropicalis Morelet en Cuba han sido alterados por prácticas inadecuadas de aprovechamiento forestal. Esta investigación tiene el objetivo de caracterizar la estructura de un bosque perturbado de Pinus tropicalis en Galalón, Pinar del Río. Para ello se realizó un inventario florístico, se determinaron los índices de valor de importancia ecológica y de importancia ecológica ampliado. Se calculó la diversidad alfa mediante el recíproco del índice de Simpson (1/D) y el índice de Jackknife, así como la diversidad beta por medio de Morisita-Horn y Jaccard. Se evaluó el grado de perturbación del área mediante indicadores de cambios en el ecosistema. Se identificaron un total 22 familias, 30 géneros y 31 especies. Los parámetros estructurales indicaron que las especies de mayor importancia ecológica son P. tropicalis, P. caribaea var. caribaea y Byrsonima crassifolia. La diversidad florística se calificó de baja y la similitud resultó en tres grupos diferenciados por su composición florística.

Palabras clave: diversidad, estructura de bosque, perturbación del hábitat.
\end{abstract}

\begin{abstract}
Natural forests of Pinus tropicalis Morelet in Cuba have been altered by inadequate management. The aim of this study was to characterize the structure of a disturbed forest of Pinus tropicalis in Galalón, Pinar del Rio. We carried out a floristic inventory, and determined the ecological importance value and the increased ecological importance index. The alpha diversity was calculated by means of the reciprocal of the Simpson index (1/D) and the Jackknife index. The beta diversity was estimated through the Morisita-Horn and Jaccard. The degree of interference of the area was evaluated by means of indicators of changes in the ecosystem. We identified 30 genera and 31 species from 22 families. The structural parameters indicated that the species of higher ecological importance were P. tropicalis, P. caribaea var. caribaea and Byrsonima crassifolia. The floristic diversity was low and the similarity indexes yielded three groups differing in floristic composition.
\end{abstract}

Key words: diversity, forest structure, habitat disturbance.

$\mathbf{E}_{p}^{\mathrm{n}}$ Cuba existen tres especies de pino y una variedad: Pinus tropicalis Morelet, P. caribaea Morelet var. caribaea, P. cubensis Griseb. y P. maestrensis Bisse (López, 1982). Las últimas dos especies forman bosques abiertos en áreas de bajas elevaciones, mientras que $P$. tropicalis y $P$. caribaea var. caribaea forman bosques extensos en zonas montañosas del oeste del país (Bisse, 1988).

Pinus tropicalis, llamado pino blanco o pino hembra, es una especie que representa un relicto del linaje eurasiático de los pinos Diploxylon (sección Pinus) en Norteamérica (Gernandt et al., 2005). Se distribuye en Pinar del Río y en la Isla de la Juventud, en las cimas de los cerros y montañas,

principalmente en áreas con alta recurrencia de incendios (Samek et al., 1969; De las Heras et al., 2005). Esta especie está adaptada a los fuegos, los árboles adultos dejan caer sus acículas, lo que produce una gran cantidad de material combustible en el suelo, ello promueve la rápida extensión de los incendios (Bisse, 1988). El fuego afecta la estructura de la vegetación, la composición florística y las características del suelo de los bosques de P. tropicalis (De las Heras et al., 2005).

La superficie que ocupan los bosques de la especie estudiada, dentro de Pinar del Río, es cada vez menor y las poblaciones en Isla de la Juventud están muy disminuidas. 


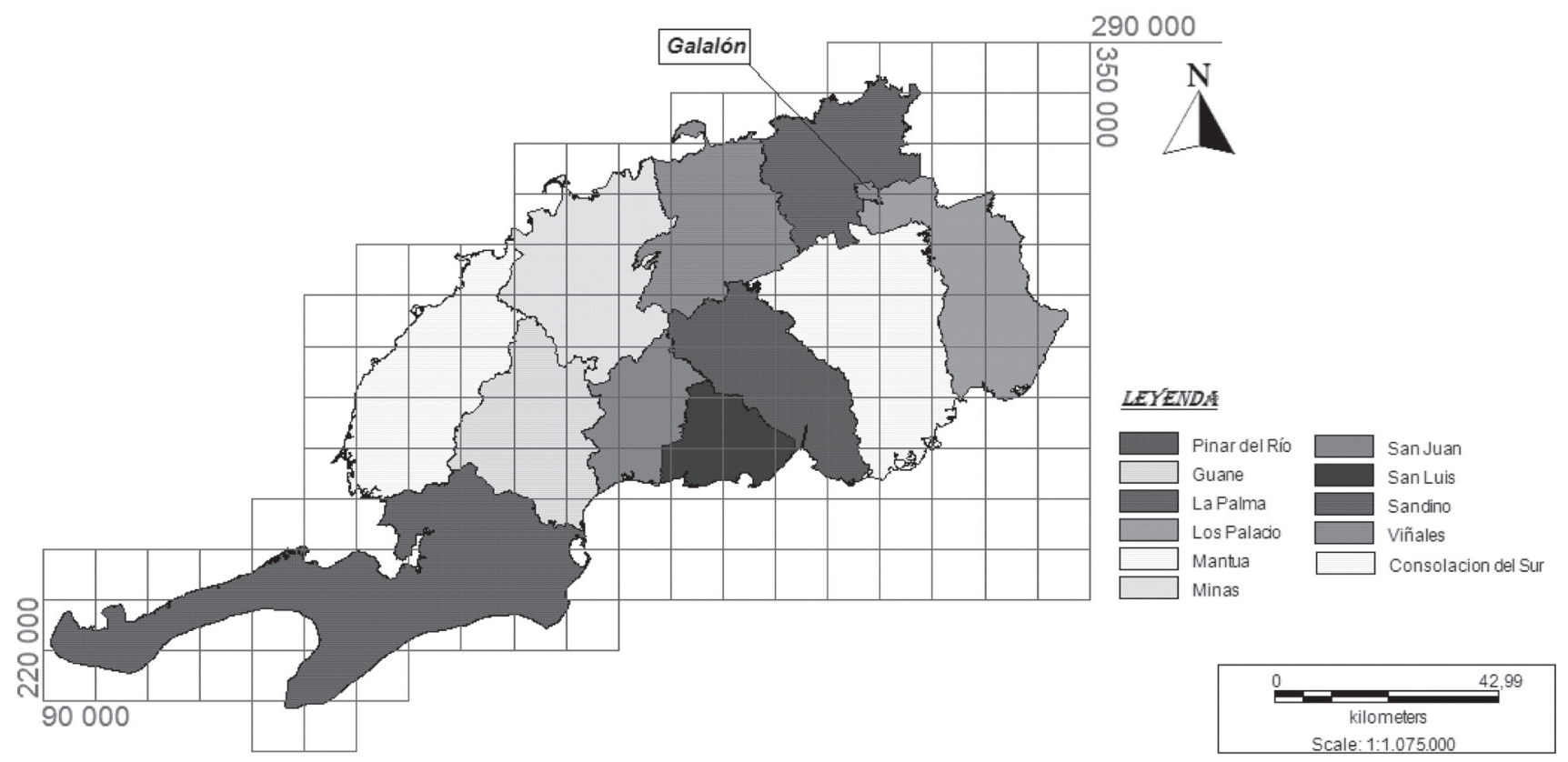

Figura 1. Ubicación geográfica del área de estudio, bosque de Pinus tropicalis en Galalón, Pinar del Río, Cuba.

Históricamente, esta especie se distribuía en la parte norte de Pinar del Río a baja elevación, pero en la actualidad sólo se encuentra en discretas poblaciones localizadas en áreas montañosas en el noreste, centro y noroeste, aunque en esta última zona las poblaciones parecen ser más continuas (Geada, 2003). Recientemente, se ha mostrado un interés por Pinus tropicalis por parte de las instituciones forestales del país debido, principalmente, a su importancia económica (Álvarez, 2004). En la actualidad, existen rodales de esta especie donde el manejo forestal se realiza por el método de talas selectivas y en otros se hacen quemas prescritas, pero se desconoce el efecto que causan estos tipos de manejo en la estructura de la vegetación. Según O’Brien et al. (2008), al removerse las copas de los árboles de esta especie adaptada a los incendios, se altera la continuidad de la materia orgánica en el suelo que sirve como combustible, por lo que pueden ocurrir cambios en la estructura y composición de la vegetación.

En Cuba, las perturbaciones realizadas a diversos hábitats naturales debido a su uso han afectado negativamente a la diversidad biológica (Febles, 2009) y probablemente la estructura de la vegetación. Esta investigación tiene el objetivo de caracterizar la estructura de un bosque perturbado de Pinus tropicalis en Galalón, Pinar del Río, Cuba, a partir de sitios con diferente historial de manejo.

\section{Materiales y métodos}

Área de estudio. El área de estudio se localiza en un bosque natural de Pinus tropicalis perteneciente a la localidad de Galalón, ubicada en el municipio La Palma, provincia de
Pinar del Río, Cuba. Limita al norte con Cajálbana, al sur con Los Palacios, hacia el este con La Güira y al oeste con Juan Manuel. El área está ubicada entre las coordenadas $22^{\circ}$ $11^{\prime} 780^{\prime \prime}$ y $22^{\circ} 13^{\prime} 100^{\prime \prime}$ de latitud norte, $84^{\circ} 03^{\prime} 820^{\prime \prime}$ y $84^{\circ}$ $05^{\prime} 130$ " de longitud oeste. Para su localización se utilizó el mapa de cobertura actual y uso de la tierra del municipio La Palma, mediante interpretación de la base cartográfica del Grupo Empresarial GEOCUBA, utilizando el programa Mapinfo Profesional versión 8.0 (Figura 1).

Se seleccionaron seis parcelas de $400 \mathrm{~m}^{2}$ a lo largo del área del bosque natural, determinadas por medio del método de área mínima (Feinsinger, 2004). La distribución se realizó mediante el método de muestreo sistemático, al considerar la fragmentación que ha sufrido la formación natural donde se presentan áreas en forma de islas (Loetsch y Haller, 1973). Además, se consideraron criterios como orientación de los rodales, topografía del terreno y representatividad de las actividades de manejo y aprovechamiento. El esfuerzo de muestreo se validó mediante el método de la curva de riqueza de especies (Figura 2), la cual indicó que a partir de la parcela seis se encuentra el punto de inflexión o estabilización de la curva. Lo anterior permite inferir la repetición de las especies en las parcelas, y que por lo tanto, con este tamaño de muestra es suficiente, aunque pudiera aumentarse en función de la disponibilidad de tiempo y recursos, pues se trata de un bosque antropizado, donde lógicamente, pueden presentarse nuevas especies con el trascurso del tiempo.

Las parcelas 1, 2 y 3 tienen orientación sur y pertenecen a Empresa Forestal Integral La Palma, donde se realizan talas selectivas de Pinus caribaea var. caribaea; y las parcelas 4, 5 y 6 de orientación norte son patrimonio de la Unidad de 
Manejo Mil Cumbres. En las parcelas 4 y 5 se han realizado quemas prescritas a partir del año 2002 para favorecer la regeneración natural de $P$. tropicalis y la parcela 6 es considerada un área exclusiva de conservación.

Composición florística. Se realizó un inventario florístico a partir del recuento e identificación de todas las especies presentes en las parcelas de estudio donde se determinaron familias, géneros y especies.

Estructura del bosque. La estructura horizontal del bosque se obtuvo seleccionando todas las especies con diámetros a la altura del pecho $(1.3 \mathrm{~m})$ superiores a $2.5 \mathrm{~cm}$. A partir del método propuesto por Lamprecht (1990), se determinó el índice de valor de importancia ecológica (IVIE) que considera la sumatoria de la abundancia, dominancia (área basal) y frecuencia relativa. Se determinó la regeneración natural (RN) mediante la metodología propuesta por Grela (2003), como la suma de los parámetros abundancia y frecuencia de todos los individuos con altura $\leq 2 \mathrm{~m}$; y la regeneración natural relativa (RNRi), como el porcentaje de regeneración natural de cada especie respecto al total. Con los valores de RNRi y el IVIE se determinó el índice de valor de importancia ecológica ampliado (IVIEA; Finol, 1971).

Diversidad de especies. Se determinó la diversidad florística (diversidad alfa) mediante el cálculo del recíproco de Simpson (1/D; Moreno, 2001), así como la diversidad promedio de Simpson por el índice de Jackknife (Magurran, 1988; Feinsinger, 2004; Koleff, 2005). La diversidad beta se estimó mediante el índice de Morisita-Horn para comparar la similitud florística cuantitativa y el índice de Jaccard para el análisis cualitativo de presencia-ausencia de especies, los cuales permiten contribuir al mejor entendimiento de la estructura de los ensambles de especies a través del espacio
(Magurran, 1988; Koleff, 2005). Se realizó un análisis de conglomerados jerárquicos a partir del índice de similitud de Jaccard, lo cual permitió la clasificación de las parcelas por su composición florística utilizando el programa Biodiversity Professional (McAleece, 1997). Las medidas de diversidad alfa y beta se obtuvieron con el empleo del programa Bio-DAP Ecological Diversity and it's Measurement (Thomas, 1996).

Grado de perturbación o modificación. La evaluación del grado de perturbación se realizó a partir de los criterios de Matos y Ballate (2006), basado en modificaciones del método propuesto por Fernández (1994), y con adecuaciones para este trabajo respecto a la evaluación del efecto provocado por huracanes en las parcelas seleccionadas, considerando: grado de representatividad de las especies originales, grado de representatividad de los estratos originales de la vegetación, grado de cobertura vegetal y grado de modificación o perturbación. Para evaluar el grado de representatividad de los estratos originales se tuvo en cuenta la existencia de los estratos característicos en la vegetación actual (arbóreo, arbustivo, herbáceo), y la caracterización fisonómica del área en correspondencia con la composición florística representativa, así como mediciones de estos estratos en relación con la altura característica que constituyen elementos de gran valor para el diagnóstico. La cobertura vegetal se evalúo a partir del análisis del porcentaje que ocupa la vegetación, mediante la comparación de fotos aéreas y los datos obtenidos en el Sistema de Información Geográfica (SIG) Google Earth del macizo montañoso Sierra de los Órganos, de las observaciones directas realizadas en el área de estudio mediante trabajo de campo y los criterios asumidos de los proyectos de ordenación forestal en cuanto a la vegetación original. Para la evaluación del grado de modificación del área se tuvo en cuenta los indicadores: área ocupada por



Figura 2. Curva de especies-área en un bosque de Pinus tropicalis en Galalón, Pinar del Río, Cuba. 
Cuadro 1. Parámetros estructurales del bosque de Pinus tropicalis de Galalón, Pinar del Río, Cuba.

\begin{tabular}{llll}
\hline Especies & IVIE & RNRi & IVIEA \\
\hline Pinus tropicalis & 31.38 & 11.64 & 43.02 \\
Pinus caribaea var. caribaea & 15.39 & 11.09 & 26.48 \\
Matayba apetala & 10.86 & 4.93 & 15.79 \\
Byrsonima crassifolia & 9.44 & 21.92 & 31.36 \\
Clusea rosea & 8.97 & 9.89 & 18.86 \\
Xylopia aromatica & 6.36 & 8.36 & 14.72 \\
Eugenia sp. & 5.36 & 1.57 & 6.93 \\
Chrysobalanus icaco & 4.58 & 13.47 & 18.05 \\
Pithecellobium abovale & 3.96 & 1.57 & 5.53 \\
Didomapanax morototonii & 2.83 & 12.40 & 15.23 \\
Cinnamommum elongatum & - & 1.57 & 1.57 \\
Andira inermis & - & 1.57 & 1.57 \\
\hline
\end{tabular}

caminos, extracción de recursos vegetales, ocurrencia de incendios forestales, talas, pastoreo e incidencia de vegetación secundaria e introducida y daños ocasionados por huracanes. En cada uno de estos indicadores se utilizaron matrices que evalúan cada factor modificante para transformar las variables cualitativas a cuantitativas, mediante la asignación de valores escalares de 0 hasta 3 de acuerdo al grado de perturbación del área, considerando 0 (muy alto grado de perturbación) cuando la suma de los indicadores se encuentra entre 1-4 puntos, 1 (alto grado de perturbación) entre 5-14 puntos, 2 (medio grado de perturbación) entre 15-20 puntos y 3 (bajo grado de perturbación) cuando se encuentra por encima de 20 puntos.

\section{Resultados}

Composición florística. En el inventario florístico se identificaron un total 22 familias, 30 géneros y 31 especies, lo que resulta en un área florísticamente pobre, donde predomina $P i$ nus tropicalis asociado con P. caribaea var. caribaea. Las familias más representativas resultaron ser, en este orden: Pinaceae, Poaceae, Dillenaceae, Rubiaceae y Melastomataceae.

Estructura de bosque. En el cuadro 1 se muestran los parámetros estructurales del bosque natural determinados por el IVIE, RNNi e IVIEA. Los valores del IVIE como resultado de la dominancia, abundancia y frecuencia relativa indicaron que Pinus tropicalis ocupó la mayor importancia ecológica, seguido de P. caribaea var. caribaea y Matayba apetala. El menor valor lo tuvo Didymoponax morototonii. Las especies que presentaron mayor IVIE no coinciden con las de mayor valor de RNRi. El mayor valor de IVIEA lo presentó $P$. tropicalis, seguido de Byrsonima crassifolia y $P$. caribaea var. caribaea, resultado que difiere del IVIE dado la mayor RNRi que presentó Byrsonima crassifolia. Para las especies Andira inermis y Cinnamommum elongatum no se reportaron valores de IVIE por presentar diámetros menores de $2.5 \mathrm{~cm}$; sin embargo, fue considerada su RNRi por presentar altura mayor de $2.0 \mathrm{~cm}$, no siendo especies características de la formación de pinares.

Diversidad de especies. La diversidad alfa, expresada mediante el recíproco de Simpson, (1/D) osciló con valores de 2.1 hasta 7.4, y la diversidad promedio del estimador de Simpson por Jackknife resultó de 4.56, lo cual indica que el bosque de Pinus tropicalis puede ser calificado de baja diversidad florística. El recíproco del índice de Simpson reflejó la máxima diversidad en la parcela 4.

La similitud florística entre las parcelas resultó media de acuerdo a los valores del índice de Jaccard (33\%). El índice de similitud de Morisita-Horn mostró una asociación media, donde las parcelas 3, 4, 5 y 6 presentan mayor homogeneidad en la estructura florística con una similitud mayor del $44 \%$ (Cuadro 2).

En la figura 3 se presenta el dendrograma resultante del análisis de clasificación de las parcelas por su composición florística, el cual permitió distinguir, con un porcentaje menor del 50\% de similitud, la formación de tres grupos, uno con las parcelas 1 y 5 , otro constituido por las parcelas 2,3 y 6 , y el último con la parcela 4 , está última con una divergencia marcada en relación con las demás.

Grado de perturbación o modificación. La evaluación de la representatividad de las especies y los estratos originales en la vegetación actual se consideró alta por estar presente especies típicas de estos ecosistemas: en el arbóreo, Pinus tropicalis y P. caribaea var. caribaea; en el arbustivo, Byrsonima crassifolia, Chrysobalanus icaco, Eugenia punicifolia, Pithecellobium obovale y Xylopia aromatica; y en el herbáceo, Clidemia hirta, Conostegia xalapensis, Sorghastrum stipoides, además de lianas y epífitas como Davilla rugosa y Tillandsia fasciculata. La cobertura vegetal se evaluó como alta, al obtener que entre el 35-55\% del territorio está ocupado por la vegetación.

El grado de modificación o perturbación resultó variable en el área de estudio con calificativo de bajo para las

Cuadro 2. Valores del índice de Jaccard y Morisita-Horn de parcelas de Pinus tropicalis en Galalón, Pinar del Río, Cuba.

\begin{tabular}{llllllll}
\hline \multicolumn{7}{l}{ Jaccard } \\
& & P1 & P2 & P3 & P4 & P5 & P6 \\
& P1 & & 0.42 & 0.38 & 0.33 & 0.45 & 0.43 \\
& P2 & 0.45 & & 0.61 & 0.39 & 0.39 & 0.5 \\
Morisita-Horn & P3 & 0.09 & 0.16 & & 0.35 & 0.35 & 0.65 \\
& P4 & 0.5 & 0.44 & 0.56 & & 0.43 & 0.35 \\
& P5 & 0.23 & 0.20 & 0.63 & 0.54 & & 0.41 \\
& P6 & 0.07 & 0.24 & 0.48 & 0.38 & 0.54 & \\
\hline
\end{tabular}




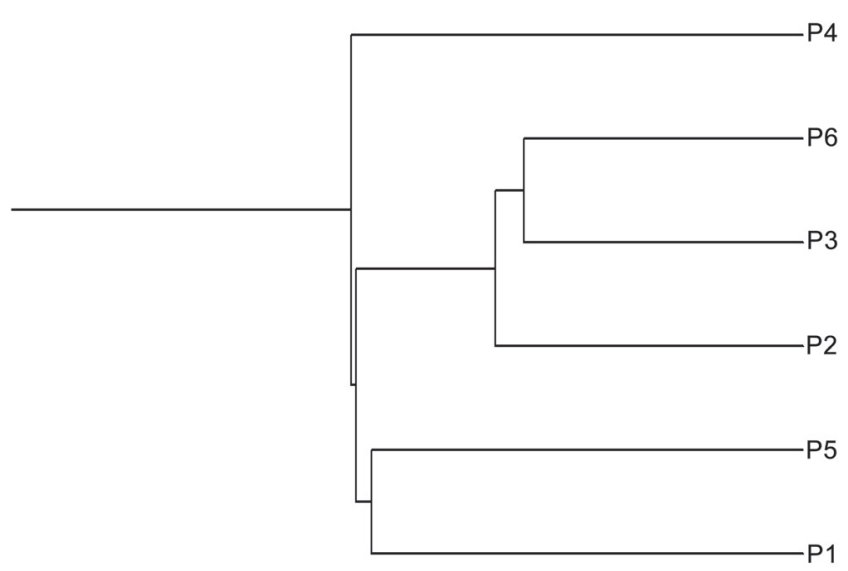

0 50

Jaccard (\% de similitud)

Figura 3. Dendrograma que muestra la clasificación de las parcelas de un bosque perturbado de Pinus tropicalis en Galalón, Pinar del Río, Cuba, de acuerdo a la composición florística.

parcelas 4 y 6 , medio en 1,3 y 5 , y alto para la parcela 2 . Se evidenció que los indicadores "área ocupada por caminos forestales" y "actividad de pastoreo" no contribuyeron a la evaluación del grado de perturbación debido a su igual comportamiento en las parcelas. Los restantes indicadores incidieron en las parcelas 1,2 y 3 de manera diferente a las 4,5 y 6 . En el caso del indicador "ocurrencia de fuegos", se considera de efecto moderado para las parcelas 4 y 5 por ser un área donde se realizan quemas prescritas de forma dirigida y controlada, en cuanto a las "talas" se tuvo en cuenta

Cuadro 3. Matriz de evaluación del grado de perturbación del bosque de Pinus tropicalis de Galalón, Pinar del Río, Cuba. medio (M), alto (A), bajo (B).

\begin{tabular}{lllllll}
\hline Indicadores & \multicolumn{7}{c}{ Parcela } \\
& $\mathbf{1}$ & $\mathbf{2}$ & $\mathbf{3}$ & $\mathbf{4}$ & $\mathbf{5}$ & $\mathbf{6}$ \\
\hline Área ocupada por caminos & 5 & 5 & 5 & 5 & 5 & 5 \\
$\quad \begin{array}{l}\text { Grado de extracción de } \\
\quad 2\end{array}$ & 2 & 2 & 3 & 3 & 3 \\
$\quad$ recursos vegetales & & & & & & \\
Ocurrencia de fuegos & 3 & 3 & 3 & 2 & 2 & 3 \\
Grado de tala & 0 & 0 & 1 & 3 & 3 & 3 \\
Actividad de pastoreo & 3 & 3 & 3 & 3 & 3 & 3 \\
Incidencia de la vegetación & 2 & 1 & 1 & 3 & 2 & 3 \\
$\quad$ secundaria e introducida & & & & & & \\
$\quad \begin{array}{l}\text { Daños por huracanes } \\
\text { Grado de perturbación }\end{array}$ & 0 & 0 & 1 & 2 & 2 & 3 \\
$\quad 15$ & 14 & 16 & 21 & 20 & 23 \\
$\quad$ Suma de indicadores) & & & & & & \\
$\quad$ de perturbación & $\mathrm{M}$ & $\mathrm{A}$ & $\mathrm{M}$ & $\mathrm{B}$ & $\mathrm{M}$ & $\mathrm{B}$ \\
\hline
\end{tabular}

las consecuencias devastadoras provocadas por las brigadas de aprovechamiento posterior al paso de los huracanes. La puntuación diferenciada por daños ocasionados por huracanes afectaron más a las parcelas 1,2 y 3 , debido a su orientación geográfica con respecto a la trayectoria de estos y por presentar menor densidad en el estrato superior, todo lo anterior explica los criterios para la calificación otorgada (Cuadro 3).

\section{Discusión}

Composición, estructura y diversidad florística. Los resultados de la composición florística coinciden con lo señalado por Samek y del Risco (1989), al referir a los pinares de Alturas de Pizarras como un distrito florísticamente pobre y un centro antiguo de evolución de esta formación boscosa. Asimismo, existe coincidencia con las especies reportadas para estos ecosistemas por los autores mencionados anteriormente, así como por Capote y Berazaín (1984).

La mayor importancia ecológica de las especies Pinus tropicalis y $P$. caribaea var. caribaea está dada por presentar la mayor dominancia, son las de máximo valor económico y de conservación por tratarse de especies endémicas con prioridades y algún grado de amenaza. Sin embargo, los resultados del IVIE de estas especies no son muy distantes del resto, lo que es un reflejo de la antropización a la cual están siendo sometidas.

La especie Matayba apetala ocupa un índice de importancia ecológica relevante, lo cual pudiera deberse a la modificación en la estructura del bosque producto de la actividad antrópica. Esto corrobora la hipótesis de perturbación intermedia de Connell (1978), que postula que la apertura de claros en el bosque y la capacidad de ciertas especies de prosperar en ellos, favorecen un nivel de diversidad mucho más alto (a escala local y regional) que si faltaran esas perturbaciones.

Alanís-Rodríguez et al. (2010) refieren que IVIEA es el indicador más importante para evaluar la dinámica del bosque, debido a la integración que hace de la estructura horizontal y vertical, tanto de la masa adulta como de la regeneración natural. En este estudio, la especie Pinus tropicalis sigue siendo de mayor importancia por el valor de IVIEA; sin embargo, Byrsonima crassifolia cobra significancia dada la presencia de una mayor regeneración natural. De acuerdo a lo reportado por Bonilla et al. (2009), $B$. crassifolia es una especie de importancia ecológica en la asociación con los pinos con una alta capacidad de rebrote después de la ocurrencia de incendios. También los valores altos reportados para $P$. caribaea var. caribaea indican que al parecer se mantendrá la composición mixta de la formación del pinar.

El índice de Simpson, que considera la dominancia de las especies, es comúnmente preferido por los ecólogos, pues ofrece información acerca de la probabilidad que dos 
individuos extraídos al azar pertenezcan a diferentes especies, siendo menos sensible a la riqueza de especies (Hurlbert, 1971). La baja diversidad obtenida por el recíproco de Simpson pudiera atribuirse a la dominancia de las especies presentes en la formación de pinar que condiciona esta característica. Valdés et al. (2008) reportan valores similares en la diversidad para los bosques de pinos de la región norte de este territorio.

La mayor diversidad presentada por la parcela 4 está determinada por la mayor abundancia de especies. Este sitio corresponde a un área donde se realizan quemas prescritas, práctica que según Bonilla et al. (2009) favorece la regeneración de especies y crea condiciones particulares para la presencia de otras.

Los niveles de similitud entre las unidades de muestreo están asociados a la presencia exclusiva de especies, Dactyloctenium argyptium se encuentra únicamente en las parcelas 1 y 5; Chrysobalanus icaco, Distictis anaphelanthala y Pinus caribaea var. caribaea en las parcelas 2, 3 y 6; y Tabernaemontana citrifolia y Tillandsia fasciculata en la parcela 4 . Este agrupamiento refleja que la composición florística y la estructura del bosque están determinadas por los factores antrópicos y las prácticas de aprovechamiento forestal.

Grado de perturbación o modificación. La perturbación de la vegetación, provocada por factores antrópicos, altera sensiblemente las comunidades (Maass et al., 2010; De la O-Toris et al., 2012). Las parcelas que presentaron medio y alto grado de perturbación en el bosque de Pinus tropicalis se debe fundamentalmente a la incidencia de huracanes y prácticas inadecuadas de aprovechamiento forestal, lo que posibilita la presencia de la vegetación secundaria, tal es el caso de la parcela 3 caracterizada por el predominio de $\mathrm{Ma}$ tayba apetala, especie representativa de bosques secundarios e indicadora de sitios más perturbados por la explotación forestal (González et al., 2011). Hobbs y Huenneke (1992), aseguran que cualquier cambio en el régimen histórico de disturbio de un ecosistema puede alterar la composición de especies mediante la disminución de la importancia de las especies nativas, la creación de oportunidades para el ingreso de especies exóticas o por ambos medios. Los sitios donde el nivel de perturbación es mayor se caracterizan por el elevado número de individuos con diámetros pequeños, lo que le confiere al bosque una elevada densidad (Jiménez, 2012).

\section{Conclusiones}

El bosque de Pinus tropicalis en Galalón es pobre florísticamente. Las especies de mayor importancia ecológica son P. tropicalis, P. caribaea var. caribaea y Byrsonima crassifolia. Su grado de perturbación resultó entre medio y bajo. Los huracanes y el aprovechamiento inadecuado son las causas fundamentales del deterioro.

\section{Literatura citada}

Alanís-Rodríguez E., Jiménez-Pérez J., Pando-Moreno M., Aguirre-Calderón O., Treviño-Garza E.J., y García-Galindo P.C. 2010. Efecto de la restauración ecológica post-incendio en la diversidad y estructura del componente arbóreo del Parque Ecológico Chipinque, México. Madera y Bosques 16:39-54.

Álvarez J. 2004. Transformations in Cuban Agriculture after 1959. EDIS document FE481. Department of Food and Resource Economics, Florida Cooperative Extension Service. University of Florida, Gainesville.

Bisse J. 1988. Árboles de Cuba. Editorial Científico-Técnica, La Habana.

Bonilla V.M., Valdés L. y Martínez B.L.W. 2009. Regeneración natural de Pinus tropicalis Morelet y vegetación asociada después de un incendio. Revista Cerne 15:215-220.

Capote R.P. y Berazaín R. 1984. Clasificación de las formaciones vegetales de Cuba. Revista Jardín Botánico Nacional 5:27-75.

Connell J.H. 1978. Diversity in tropical rain forests and coral reefs. Science 199: 1302-1310.

De la O-Toris J., Maldonado B. y Martínez-Garza C. 2012. Efecto de la perturbación en la comunidad de herbáceas nativas y ruderales de una selva estacional mexicana. Botanical Sciences 90:469-480.

De las Heras J., Bonilla M. y Martínez L.W. 2005. Early vegetation dynamics of Pinus tropicalis Morelet forests after experimental fire (W Cuba). Annals of Forest Science 62:771-777.

Febles G. 2009. La diversidad biológica en Cuba, características y situación actual. Estrategia nacional y plan de acción. Revista Cubana de Ciencias Agrícolas 43:211-223.

Feinsinger P. 2004. El Diseño de Estudios de Campo para la Conservación de la Biodiversidad. Editorial FAN, Santa Cruz de la Sierra.

Fernández M.C. 1994. Consideraciones teórico-metodológicas acerca de la evaluación de la cubierta vegetal en el medio ambiente. En: Arcia R.M.I. Ed. Geografía del Medio Ambiente: Una Alternativa del Ordenamiento Ecológico. Colección Ciencias y Técnicas Núm. 24, pp. 96-103, Universidad Autónoma del Estado de México, Toluca.

Finol H. 1971. Nuevos parámetros a considerarse en el análisis estructural de las selvas vírgenes tropicales. Revista Forestal Venezolana 21:29-42.

Geada L.G. 2003. Phylogenetic relationships of Diploxylon Pines, genetic variation of Pinus tropicalis and the Cuban pines. Tesis Doctoral. Forest Resource Biology. Universidad de Ehime, Japón. 80 pp.

Gernandt D.S., Geada L.G., Ortiz G.S. y Liston A. 2005. Phylogeny and classification of Pinus. Taxon 54:29-42.

González D.S., León S.M.A., Acosta D.J., Pedroso G.E. y Calderín M.C. 2011. La deforestación del bosque siempre verde en Soroa Candelaria, Artemisa, Cuba. Revista Facultad Nacional de Agronomía-Medellín 64:5937-5947.

Grela I.A. 2003. Evaluación del estado sucesional de un bosque subtropical de quebradas en el norte de Uruguay. Acta Botanica Brasilica 17:315-324.

Hobbs R.J. y Huenneke L.F. 1992. Disturbance, diversity, and invasion: implications for conservation. Conservation Biology 6:324-337.

Hurlbert S.H. 1971. The nonconcept of species diversity: a critique and alternative parameters. Ecology 52:577-86. 
Jiménez A.G. 2012. Contribución a la ecología del bosque semideciduo mesófilo en el sector de la Reserva de la Biosfera "Sierra del Rosario" orientada a su conservación. Tesis Doctoral en Ciencias Forestales. Universidad de Pinar del Río. Pinar del Río, Cuba. 107 pp.

Koleff P. 2005. Conceptos y medidas de la diversidad beta. En: Halffter G., Soberón J., Koleff P. y Melic A. Eds. Sobre Diversidad Biológica: el Significado de las Diversidades Alfa, Beta y Gamma, pp. 19-40, Monografías 3er Milenio, Zaragoza

Lamprecht H. 1990. Silvicultura en los trópicos. Los ecosistemas forestales en los bosques tropicales y sus especies arbóreas. Posibilidades y métodos para un aprovechamiento sostenido. Deutsche Gesellschaft für. Technische Zusammenarbeit (GTZ) GmbH., Eschborn.

Loetsch F. y Haller K.E. 1973. Forest Inventory. Vol. I. BLV Verlags gesellschaft, Munich.

López A. 1982. Variabilidad del género Pinus (Pinaceae) en Cuba. Acta Botánica Cubana 12:1-43.

Maass M., Búrquez A., Trejo I., Valenzuela D., González M.A., Rodríguez M. y Arias H. 2010. Amenazas. En: Ceballos G., Martínez L., García A., Espinoza E., Bezaury C.J. y Dirzo R. Eds. Diversidad, Amenazas y Áreas Prioritarias para la Conservación de las Selvas Secas del Pacífico de México, pp. 321-346, Fondo de Cultura Económica, Comisión Nacional para el Conocimiento y Uso de la Biodiversidad, México D.F.

Recibido: 23 de julio de 2013

Aceptado: 8 de octubre de 2013
Magurran A.E. 1988. Diversidad Ecológica y su Medición. Ediciones Vedrá, Barcelona.

Matos J. y Ballate D. 2006. ABC de la Restauración Ecológica. Editorial Feijóo, Santa Clara.

McAleece N., Gage J.D.G., Lambshead P.J.D. y Paterson G.L.J. 1997. BioDiversity Professional statistics analysis software. $<$ http://www.sams.ac.uk/research/software> (consultado $20 \mathrm{de}$ enero, 2013) .

Moreno C.E. 2001. Métodos para Medir la Biodiversidad. Manuales y Tesis. SEA, Zaragoza.

O’Brien J.J., Hiers J.K., Callaham M.A.Jr., Mitchell R.J. y Jack S.B. 2008. Interactions among overstory structure, seedling life-history traits, and fire in frequently burned neotropical pine forests. AMBIO: A Journal of the Human Environment 37:542-547.

Samek V. y del Risco E. 1989. Los pinares de la provincia de Pinar del Río, Cuba. Estudio sinecológico. Academia de Ciencias de Cuba 19:13-19.

Samek V., López A. y del Risco E. 1969. Observaciones sobre la repoblación de pinos en la región de las Cañas (Macurijes), Pinar del Río. Academia de Ciencias de Cuba 5:1-16.

Thomas G. 1996. Bio-DAP Ecological Diversity and its Measurement. Fundy National Park, New Brunswick.

Valdés V.O., García F.J.M., Ramos P. y Rodríguez M. 2008. Educación, Protección Ambiental y Prevención de Desastres: Escuela, Familias y Comunidad. Editorial Pueblo y Educación, La Habana. 